\title{
Bushen Huoxue Acupuncture Inhibits NLRPI Inflammasome-Mediated Neuronal Pyroptosis in SAMP8 Mouse Model of Alzheimer's Disease
}

This article was published in the following Dove Press journal: Neuropsychiatric Disease and Treatment

\author{
Ting Zhang' \\ Bin Guan' \\ Sipin $\operatorname{Tan}^{2}$ \\ Hong Zhu' \\ Dan Ren' \\ Ruomeng $\mathrm{Li}^{1}$ \\ Lan Xiao'
}

'Department of Traditional Chinese Medicine, The Third Xiangya Hospital of Central South University, Changsha, Hunan, 410008, People's Republic of China; ${ }^{2}$ Key Laboratory of Sepsis, Translational Medicine of Hunan, Department of Pathophysiology, Xiangya School of Medicine, Central South University, Changsha, Hunan, 410008, People's Republic of China
Correspondence: Lan Xiao Department of Traditional Chinese Medicine, The Third Xiangya Hospital of Central South University, 138 Tongzipo

Road, Changsha, Hunan, 410008, People's

Republic of China

Tel +86-I5802686038

Email xiaol038@I63.com
Background: It was indicated that nucleotide-binding oligomerisation domain-like receptor protein 1 (NLRP1) inflammasome-mediated pyroptosis is involveg in the progression of Alzheimer's disease (AD). This study was designed to explore the effect of Bushen Huoxue Acupuncture on cognitive defect and NLRP1 inflammasome-mediated pyroptosis in AD mouse. Methods: Senescence-accelerated mouse prone 8 (SAMP8) mice were used as a model of AD. Bushen Huoxue Acupuncture was performed in four acupoints: "Baihui acupoint" (GV20), "Shenshu acupoint" (BL23), "Xuehai acupoint" (SP10), and "Geshu acupoint" (BL17). Morris water maze test was performed to evaluate the cognitive function of the mouse. The levels of $A \beta_{1-40}, A \beta_{1-42}$, IL-1 $\beta$, and IL-18 were examined by ELISA assay. Neuronal apoptosis and damage in hippocampal tissues were measured using TUNEL and Nissl staining, respectively. The expression of NLRP1, ASC, cleaved caspase-1, IL-1 $\beta$, and IL-18 was examined using Western blot.

Results: Bushen Huoxue Acupuncture improved the learning and memory deficits of $\mathrm{AD}$ mouse. Meanwhile, Bushen Huoxue Acupuncture decreased the production of A $\beta$ in hippocampal tissues of SAMP8 mice and attenuated the neuronal apoptosis and damage. Furthermore, Bushen Huoxue Acupuncture inhibited NLRP1 inflammasome activation in SAMP8 mice.

Conclusion: Bushen Huoxue Acupuncture could notably attenuate the cognitive defect of mouse AD model and inhibit NLRP1 inflammasome-mediated pyroptosis.

Keywords: Bushen Huoxue Acupuncture, Alzheimer's disease, NLRP1 inflammasome, pyroptosis, hippocampal neurons

\section{Introduction}

Alzheimer's disease (AD) is an age-associated neurodegenerative disorder, characterized by progressive decline in cognitive and functional skill. ${ }^{1}$ At present, there are no effective drugs for AD therapy. Pathological deposition of amyloid- $\beta$ (A $\beta)$ protein, intracellular neurofibrillary tangles, and neuronal loss caused by neuronal apoptosis are the important pathological characteristics of $\mathrm{AD} .^{2-5}$ In this present study, we explored the effect of Bushen Huoxue Acupuncture on cognitive defect of mouse AD model from a traditional Chinese medicine (TCM) point of view, and preliminarily analyzed the action mechanism of Bushen Huoxue Acupuncture in improving the disease.

Nucleotide-binding oligomerisation domain-like receptor protein 1 (NLRP1) inflammasome is a multi-protein complex, composed of NLRP1, pro-caspase-1 
and adaptor protein apoptosis-associated speck-like protein (ASC). ${ }^{6}$ In response to A $\beta$ aggregates, NRLP1 inflammasome is activated in AD. Subsequently, activated NLRP1 inflammasome induced cleaves caspase-1 into its active subunits. Next, caspase-1 activation leads to processing and maturation of the cytokines interleukin-1 $\beta$ (IL$1 \beta$ ) and interleukin-18 (IL-18), and a lytic form of cell death termed pyroptosis. ${ }^{6,7}$ It was shown that NLRP1/ caspase-1-dependent pyroptosis plays an important role in AD progression. ${ }^{6,8}$ For instance, Tan et al demonstrated increased cerebral NLRP1 levels in $\mathrm{APP}_{\mathrm{swe}} / \mathrm{PS} 1_{\mathrm{dE} 9}$ transgenic mice. NLRP1 or caspase-1 deficient mice showed significant reduction in neuronal pyroptosis and reversed cognitive impairments. ${ }^{8} \mathrm{Xu}$ et al reported that Ginsenoside $\mathrm{Rg} 1$ protect against $\mathrm{H}_{2} \mathrm{O}_{2}$-induced neuronal damage by inhibiting the activation of NLRP1 inflammasome in hippocampal neurons in vitro. ${ }^{9}$ These findings suggested that the strategies targeting NLRP1 inflammasome activation might be useful in the therapy of AD.

Acupuncture is an important TCM therapy that has been practiced in China for 4000 years. ${ }^{10}$ It is welltolerated and has quite rare adverse effects. ${ }^{11}$ Acupuncture has been known to be an effective therapeutic approach for AD treatment. Jia et al demonstrated that acupuncture could effectively enhance the learning and memory functions of patients with $\mathrm{AD} .{ }^{12}$ Cui et al reported that acupuncturing at GV24 and bilateral GB13 acupoints may enhance the cognitive function and synaptic plasticity of $\mathrm{AD}$ mouse through regulating the distribution of certain kinds. ${ }^{13}$ A growing evidence revealed that acupuncture may enhance hippocampal connectivity in $\mathrm{AD}$ patients, activate specific brain regions related to cognition, ${ }^{12}$ and improve cognitive function and global clinical status. ${ }^{12,14-16}$ However, its clinical effects and underlying mechanisms are not fully understood.

According to TCM, kidney deficiency and blood stasis are the basic pathogenic factors of $\mathrm{AD}$, and run through the whole process of $\mathrm{AD}$. Accordingly, we developed an acupuncture method of Bushen Huoxue (tonifying kidney and resolving blood stasis) in the acupoints of "Baihui acupoint" (GV20), "Shenshu acupoint" (BL23), "Xuehai acupoint" (SP10), and "Geshu acupoint" (BL17). Here, the aim of our study is to examine whether Bushen Huoxue Acupuncture alleviates the cognitive deficits, $A \beta$ aggradation, and neuronal apoptosis in senescence-accelerated mouse prone 8 (SAMP8) mice, a natural model of $\mathrm{AD}$, and the regulatory mechanism of it. ${ }^{17}$ In this present study, we demonstrated that Bushen Huoxue Acupuncture effectively improved the learning and memory functions of mouse AD model, and inhibited NLRP1 inflammasomemediated neuronal pyroptosis.

\section{Materials and Methods}

\section{Animals}

Thirty SAMP8 mice (age, 7-month old; weight, 26-30 g) and ten of age-matched senescence-accelerated mouse resistance 1 (SAMR1) mice were raised in the Experimental Animal Center of The Third Xiangya Hospital of Central South University. All animals were housed in a room with controlled temperature (20 23 ${ }^{\circ}$ C), humidity (55 58\%), and lighting (12 h light/dark cycle). All mice were provided with enough food and water. After one week of adaptation feeding, the SAMP8 mice were randomly divided into three groups $(n=10$ per group): AD model, nonacupuncture, and acupuncture. And age-matched SAMR1 mice were prepared as a normal control group. All animal experiments were conducted with the approval of the Experimental Animal Ethics Committee of The Third Xiangya Hospital of Central South University. All animal experiments were performed according to the Guideline for the Care and Use of Laboratory Animals. All protocols were performed under conditions to minimize animal suffering.

\section{Acupuncture Treatment}

Mouse acupoint location and acupuncture depth referenced the experimental acupuncture. ${ }^{18}$ Mice in the acupuncture group were intervened by Bushen Huoxue Acupuncture into the acupoints of "Baihui acupoint" (GV20), "Shenshu acupoint" (BL23), "Xuehai acupoint" (SP10) and "Geshu acupoint" (BL17). GV20 was located at the center of the parietal bone, and the needle was inserted into the epicranial aponeurosis. BL23 was located adjacent to the second lumbar vertebra. SP10 was located in the middle of the medial hind thigh, medial end of the metatarsal floor to the pubic symphysis $1 / 9$. BL17 was located in the middle of the bilateral ribs under the 7th thoracic vertebra. At GV20, the needle was inserted obliquely to a depth of 3-4 mm. At BL23 and SP10, the needle was inserted perpendicularly to 2-3 mm depth. At BL17, the needle was inserted obliquely upward at an angle of $80^{\circ}$ to $2-3 \mathrm{~mm}$ depth. For BL23, SP10 and BL17, the left side was acupunctured first, and then the right side was acupunctured the next day. Mice in the nonacupuncture control group were treated by stimulating the fixed non-point under the bilateral rib, while mice in the $\mathrm{AD}$ 
model group and normal control group were raised without special treatment but administered the stimulation of catching with the same time and the same stimulus intensity. All mice were intervened daily for 6 consecutive days, followed by one day of rest, for 8 consecutive weeks.

\section{Morris Water Maze Test}

After acupuncture treatment, the Morris water maze (MWM) test was performed to evaluate the learning and memory behaviors of animals. The water maze was a circular pool with $150 \mathrm{~cm}$ of diameter and $50 \mathrm{~cm}$ of depth. The maze was divided into four equal quadrants. A circular escape platform was placed at the second (target) quadrant and submerged approximately $1.5 \mathrm{~cm}$ below the surface of the water. Mice were placed on the platform for $15 \mathrm{~s}$. The mice were placed in the water at the other quadrants and allowed to swim freely. The time they spent reaching the underwater platform (escape latency) was recorded. If mice were unable to reach the platform within $60 \mathrm{~s}$, they were gently guided to it and left on the platform for $15 \mathrm{~s}$ and the escape latency was recorded as $60 \mathrm{~s}$. After the completion of spatial navigation trial, the hidden platform was removed. Mice were placed in the pool at the location opposite to the platform position and allowed to swim freely for $60 \mathrm{~s}$. The time spent in the target quadrant and the number of crossing the original platform were recorded. All mice were approved underwent three trials per day from different release position. The MWM tests were performed once a day for six days.

\section{Enzyme-Linked Immunosorbent Assay (ELISA)}

After MWM test, all mice were euthanized by carbon dioxide. Hippocampus tissues were harvested, and then homogenized with ice-cold RIPA lysis buffer. Next, the levels of $A \beta 1-40, A \beta 1-42$, IL-1 $\beta$, and IL-18 in homogenates of hippocampus tissues were measured using a Mouse A $\beta 1-40$ ELISA Kit, Mouse A $\beta 1-42$ ELISA kit, Mouse IL-1 $\beta$ ELISA Kit, and Mouse IL-18 ELISA Kit, respectively. All ELISA assays were performed strictly according to the manufacturer's instruction of kits. Mouse A $\beta 1-40$ ELISA Kit and Mouse A $\beta 1-42$ ELISA kit were obtained from Shanghai Enzyme-linked Biotechnology (Shanghai, China). Mouse IL-1 $\beta$ ELISA Kit and Mouse IL-18 ELISA Kit were purchased from Elabscience (Houston, TX, USA).

\section{TUNEL Staining}

Hippocampal tissues were removed from the brains of mice and subsequently washed with ice-cold normal saline. After that, hippocampal tissues were fixed with $4 \%$ paraformaldehyde at $4{ }^{\circ} \mathrm{C}$ overnight. Next, all hippocampal tissues were embedded in paraffin, and sectioned at 6 $\mu \mathrm{m}$ thickness. The sections were dewaxed with xylene, and rehydrated with a graded alcohol. Neuronal apoptosis in hippocampus tissues was assessed using a TUNEL Staining Kit (Keygen Biotech, Nanjing, China) according to the manufacture's protocol. ${ }^{19}$ Finally, the total number of TUNEL-positive neuron (apoptotic neuron) in hippocampus was counted under a fluorescence microscope (Olympus Corporation, Tokyo, Japan). Randomly three different fields were selected from each section.

\section{Nissl Staining}

Hippocampal sections were stained with $1 \%$ toluidine blue for $40 \mathrm{~min}$ to stain the Nissl body in neurons. Subsequently, the sections were washed with distilled water for $5 \mathrm{~min}$, and then quickly dehydrated in graded alcohol. After that, xylene was then added and the slices were sealed using neutral gum. At last, the sections were examined under a microscope (Nikon Eclipse E100; Nikon Inc., Tokyo, Japan). The number of staining cells was counted at $400 \times$ magnification in a blinded manner. ${ }^{20}$

\section{Western Blot}

Total protein samples were extracted from the hippocampus in RIPA lysis buffer (Beyotime, Shanghai, China). Then, the protein samples were loaded and separated with $10 \%$ sodium dodecyl sulfate-polyacrylamide gel electrophoresis gels, and transferred to polyvinylidene difluoride membrane. After blocking with $5 \%$ non-fat milk for $1 \mathrm{~h}$, the membranes were incubated with primary antibodies as follows (Abcam, Cambridge, MA, USA): anti-NLRP1 (1:1,000), anti-ASC $(1: 1,000)$, anti-cleaved caspase-1 $(1: 1,000)$, anti-IL-1 $\beta$ $(1: 1,000)$, anti-IL-18 (1:1,000), and anti- $\beta$-actin $(1: 1,000)$. Then, the membranes were incubated with horseradish peroxidase-conjugated secondary antibodies (Abcam). An enhanced chemiluminescence kit (Pierce, Rockford, IL, USA) was used to develop blots. $\beta$-actin was used as the loading control.

\section{Statistical Analysis}

Statistical analysis was performed using SPSS 22.0. The unpaired Student's $t$-test and one-way analysis of variance 
were used to analyze differences between two or more groups, respectively. The analysis of escape latency in each group were analyzed using repeated measures analysis of variance. $P$ values $<0.05$ were considered significant.

\section{Results}

\section{Bushen Huoxue Acupuncture Improved Cognitive Deficit and Reduced $A \beta$ Level in $A D$ Mice}

Compared with the control group, the mice in the AD model group exhibited longer escape latency, less time spent in the quadrant that previously contained the platform (target quadrant), as well as a smaller number of platform location crosses. Importantly, compared with the $\mathrm{AD}$ or nonacupuncture group, the $\mathrm{AD}$ mice in the acupuncture group showed shorter escape latency. Furthermore, AD mice that received Bushen Huoxue Acupuncture treatment remained in the original platform quadrant longer and crossed the target quadrant more frequently than mice in the untreated $\mathrm{AD}$ group (Figure 1A).
Furthermore, the production of $A \beta_{1-40}$ and $A \beta_{1-42}$ were markedly promoted in hippocampus tissues of $\mathrm{AD}$ mice compared with the mice in the control group. Bushen Huoxue Acupuncture treatment significantly decreased the $\mathrm{AD}$ induced levels of $A \beta_{1-40}$ and $A \beta_{1-42}$ (Figure 1B).

\section{Bushen Huoxue Acupuncture Attenuated Hippocampal Neuronal Apoptosis and Damage in AD Mice}

Next, we assessed neuronal apoptosis in the hippocampus tissues in each group. Compared with the control group, the mice in the $\mathrm{AD}$ model group showed a significantly increased number of TUNEL-positive cells. Bushen Huoxue Acupuncture noticeably decreased the number of apoptotic cells (Figure 2A). Furthermore, neuronal damage was assessed histologically using Nissl staining. Nissl staining revealed significantly reduced Nissl-positive viable neuronal counts in $\mathrm{AD}$ mice, and treatment of Bushen Huoxue Acupuncture preserved neurons from
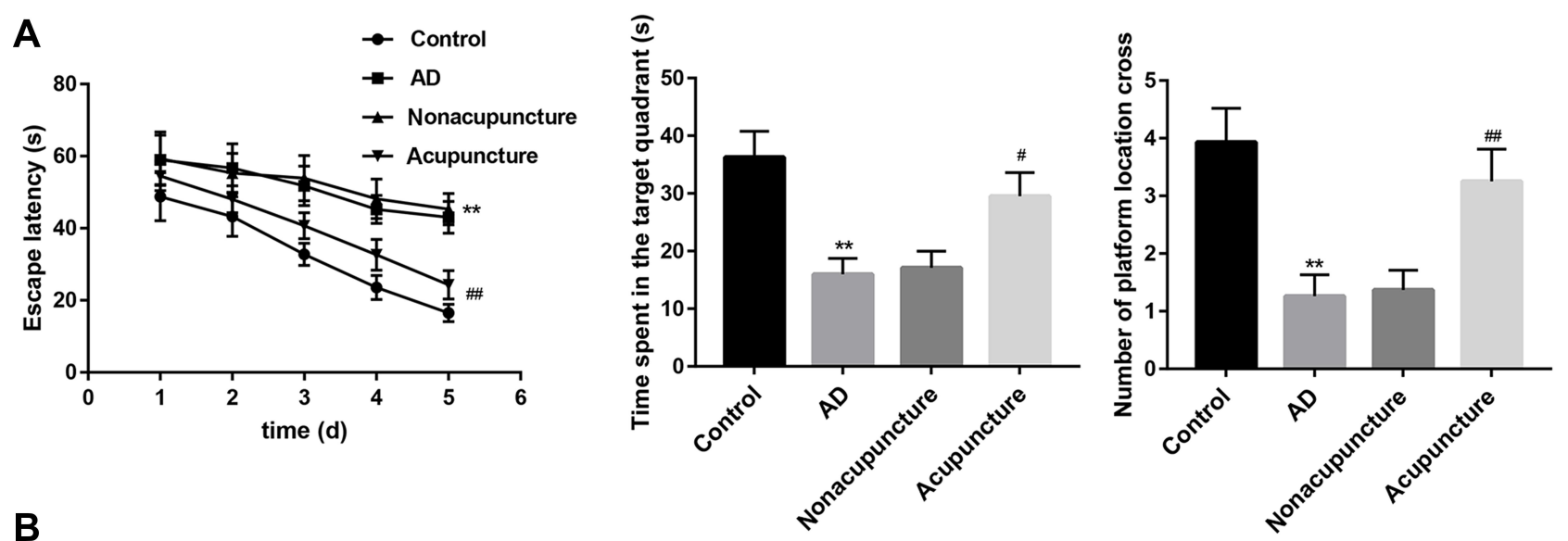

B
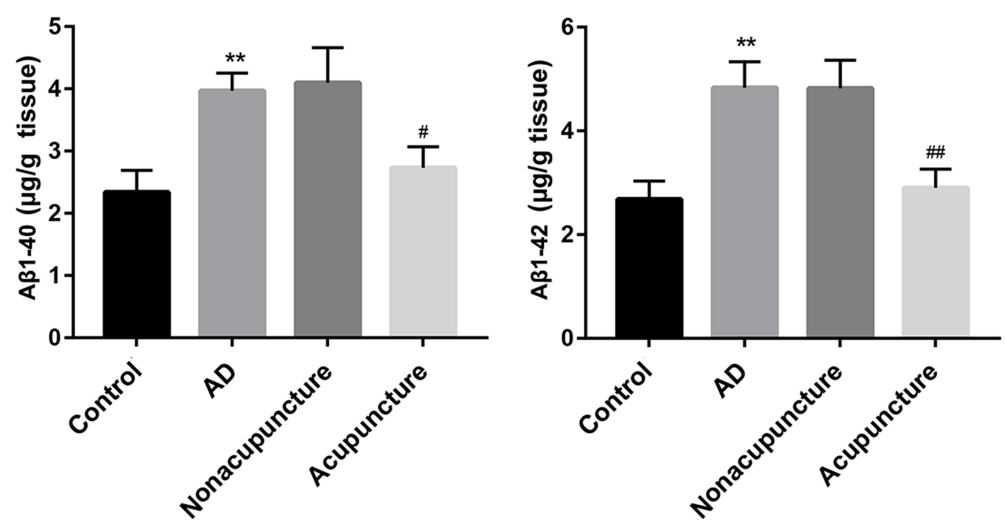

Figure I Bushen Huoxue Acupuncture improved cognitive deficits and decreased A $\beta$ level in AD mice. After the completion of Bushen Huoxue Acupuncture treatment, (A) MWM test was performed to evaluate cognitive function. The escape latency, the time spent in the target quadrant and the number of crossing the original platform were recorded. (B) Levels of $A \beta_{I-40}$ and $A \beta_{I-42}$ in homogenates from hippocampus were measured using ELISA. $N=10$ in each group. ${ }^{* *} P<0.0 I$ vs control group; ${ }^{\#} P<0.05$, and ${ }^{\#} P<0.01$ vs nonacupuncture group. 
damage, including neuron loss and degeneration (Figure 2B).

\section{Bushen Huoxue Acupuncture Inhibited NLRPI Inflammasome-Mediated Pyroptosis in AD Mice}

Compared with the control group, the expression of NLRP1, ASC, cleaved caspase-1, IL-1 $\beta$, and IL-18 were significantly upregulated in hippocampus tissues of $\mathrm{AD}$ mice (Figure 3A). Furthermore, ELISA results further confirmed the increased levels of IL-1 $\beta$, and IL-18 in the hippocampus (Figure 3B). These data indicated NLRP1 inflammasome was activated in $\mathrm{AD}$ mice. Of note, treatment with Bushen Huoxue Acupuncture notably inhibited the NLRP1, ASC, cleaved caspase-1, IL-1 $\beta$, and IL-18 expression in the hippocampus of $\mathrm{AD}$ mice (Figure $3 \mathrm{~A}$ and $\mathrm{B}$ ).

\section{Discussion}

In the current study, Bushen Huoxue Acupuncture was performed in four acupoints of "Baihui", "Shenshu", "Xuehai", and "Geshu" by manual acupuncture. "Baihui" has long been used to treat cognitive impairment clinically. ${ }^{19,21}$ "Shenshu" has the ability of strengthening kidney and dredging meridian and is related to brain function. ${ }^{20}$ "Geshu" has the effect of promoting blood circulation, and it is often compatible with "Xuehai" to treat a variety of blood stasis syndromes. ${ }^{22}$ However, the efficacy of acupuncture at the acupoints of "Xuehai" and "Geshu" for AD remains not fully understood. In this present study, Acupuncture into the four acupoints was observed to alleviate cognitive deficits, enhance degradation of $A \beta$, and attenuate neuronal apoptosis and loss in AD mice. Our results confirmed the potential of acupuncture at the acupoints of "Baihui", "Shenshu", "Xuehai", and "Geshu" for $\mathrm{AD}$ treatment.

$\mathrm{AD}$ is a neurodegenerative disorder characterized by progressive deterioration of memory, cognition, and behavioral patterns. ${ }^{21}$ Here, SAMP8 mice showed serious impairments in learning and memory functions. Importantly, Bushen Huoxue Acupuncture improved the cognitive deficit in SAMP8 mice. Excessive accumulation of $A \beta$ constitutes the central process in the pathology of $A D .^{22-25}$ The most abundant forms of $A \beta$ are $A \beta_{1-40}$ and $A \beta_{1-42}$. $A \beta_{1-40}$ forms fibril deposits that damage the cerebral vessel walls, while $\mathrm{A} \beta_{1-42}$ peptide is pathogenic by the formation of toxic fibrillation intermediates. ${ }^{26}$ Here, Bushen Huoxue Acupuncture

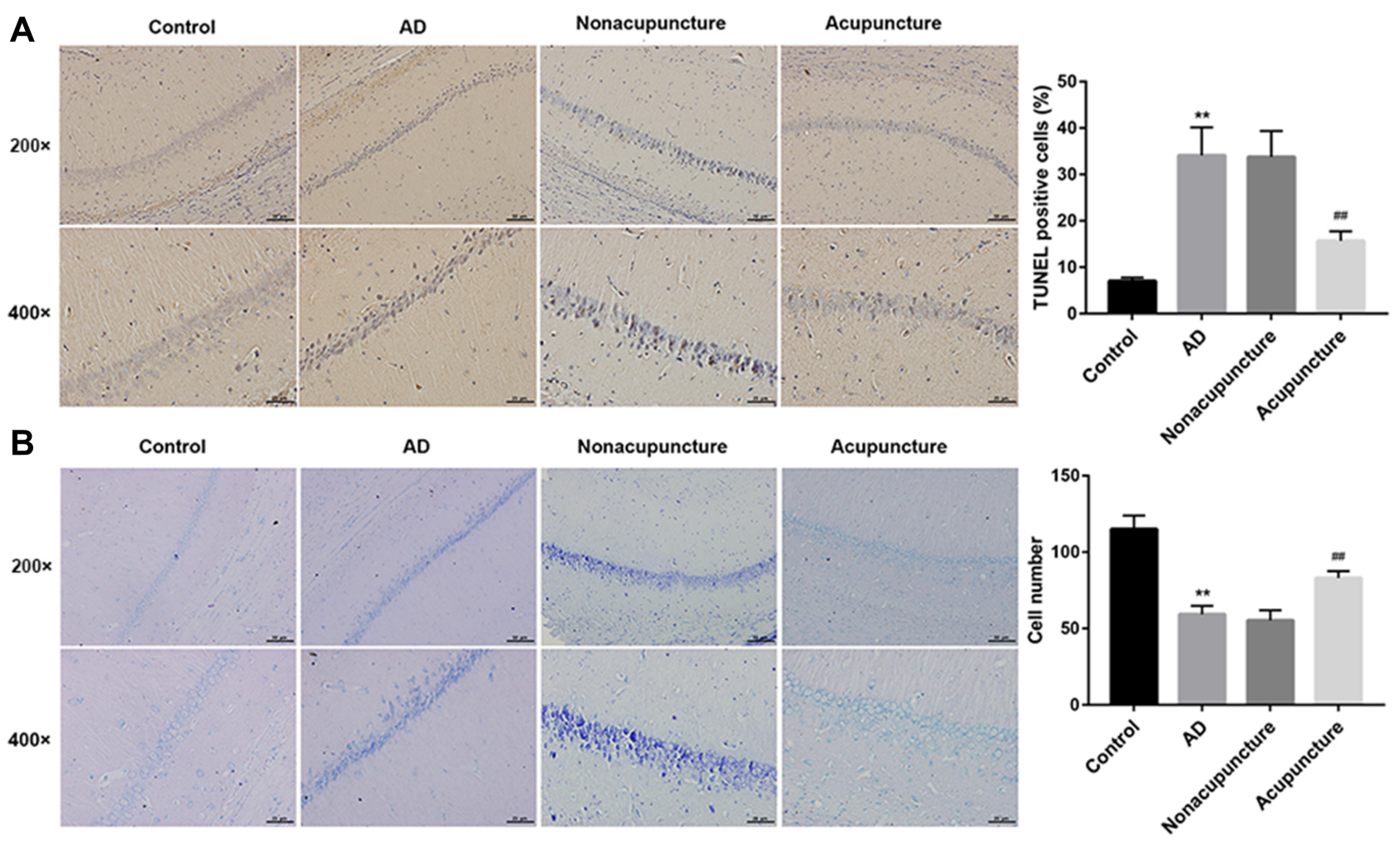

Figure 2 Bushen Huoxue Acupuncture attenuated hippocampal neuronal apoptosis and damage in AD mice. (A) Representative slides of TUNEL staining assessing hippocampal neuronal apoptosis. TUNEL-positive cells were qualified. (B) Representative slides of Nissl staining assessing hippocampal neuronal damage. Nissl-positive viable neuronal cells were counted. $\mathrm{N}=10$ in each group. ${ }^{* *} p<0.01$ vs control group; ${ }^{\#} P<0.01$ vs nonacupuncture group. 


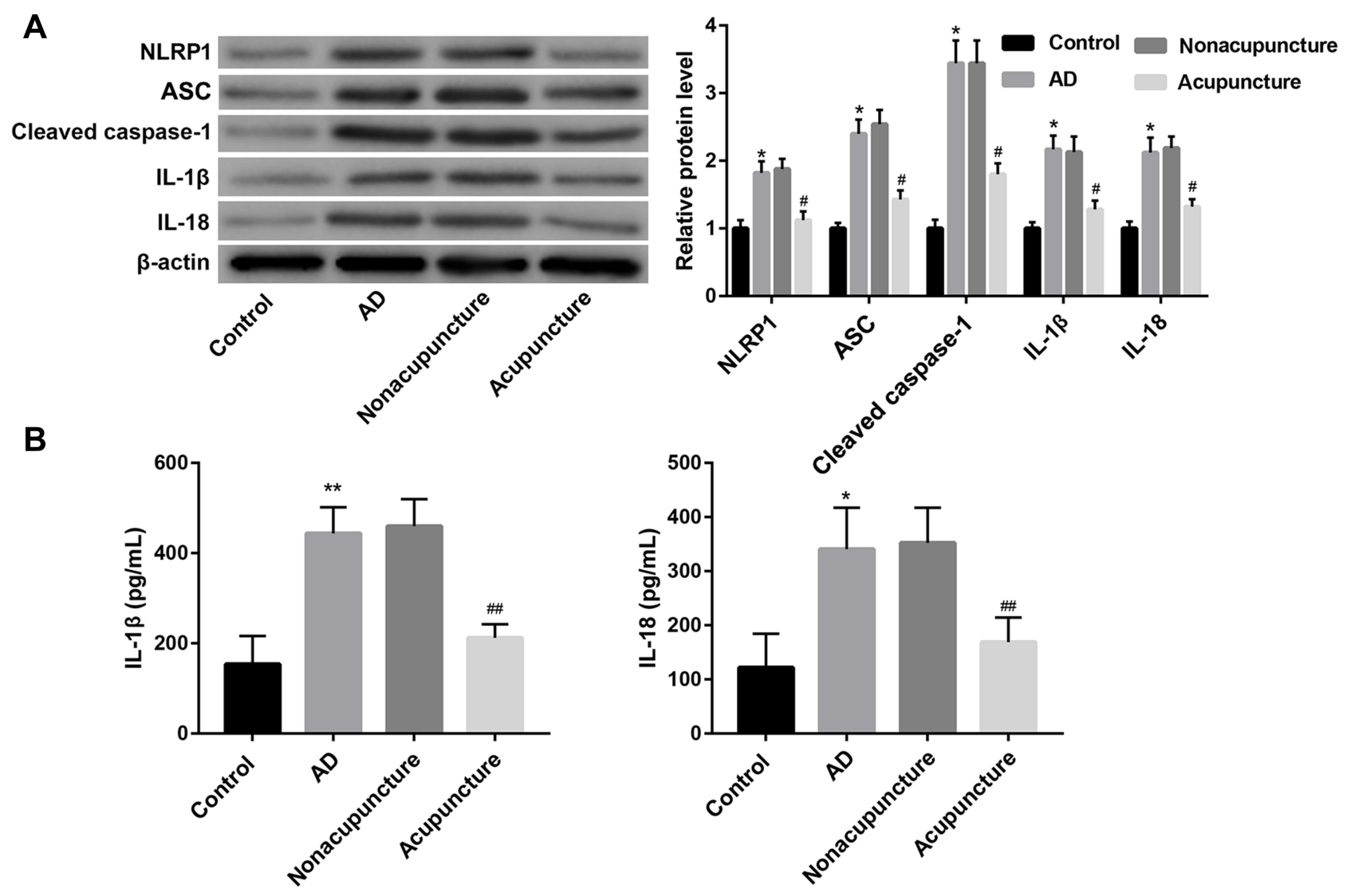

Figure 3 Bushen Huoxue Acupuncture inhibited NLRPI inflammasome-signalling pathway in AD mice. (A) The protein levels of NLRPI, ASC, cleaved caspase-I, IL-I $\beta$, and IL- 18 in the hippocampus were examined by Western blot. (B) The levels of IL-I $\beta$ and IL- I 8 in homogenates from hippocampus were measured using ELISA. $N=I 0$ in each group. $* P<0.05$, and $* * P<0.01$ vs control group; ${ }^{\#} P<0.05$, and ${ }^{\# \#} P<0.01$ vs nonacupuncture group.

could reduce the production of $A \beta_{1-40}$ and $A \beta_{1-42}$ in hippocampal tissues of $\mathrm{AD}$ mice. Neuronal apoptosis or loss is ultimately the main cause of irreversible progression of $\mathrm{AD}^{27}$ In the present study, the increased hippocampal neuronal apoptosis and damage were attenuated by intervention with Bushen Huoxue Acupuncture in AD mice. Collectively, Bushen Huoxue Acupuncture is considered to be an effective method for treatment of the cognitive impairments in AD model SAMP8 mice. However, acupuncture at a non-acupoint revealed similar results to the SAMP8 mice.

NLRP1 is highly expressed in the brain, especially in neurons and oligodendrocytes. NLRP1 inflammasome was found to be activated in the brain of AD patients. ${ }^{24,25}$ Chronic glucocorticoid exposure significantly activated NLRP1 inflammasome in hippocampal neurons, thus to increase neuron-inflammation. ${ }^{28}$ Ginsenoside Rg1 protected against neuronal degeneration by inhibiting NLRP1 inflammasome both in $\mathrm{H}_{2} \mathrm{O}_{2}$-treated hippocampal neurons in vitro ${ }^{9}$ and in dexamethasone-treated mice. ${ }^{29}$ NLRP1 inflammasome activation was proved to result in pyroptosis, a caspase- 1-dependent inflammatory form of cell death that is distinct from apoptosis or other cell death mechanisms. ${ }^{30}$ NLRP1 inflammasome assembly leads to caspase- 1 activation, which in turn drives maturation and secretion of IL-1 $\beta$ and IL-18, and induces pyroptosis. ${ }^{6,7}$ Recent studies suggested that NLRP1/caspase-1-dependent pyroptosis plays an important role in $\mathrm{AD}$ progression. ${ }^{6,8}$ Here, we found that Bushen Huoxue Acupuncture inhibited NLRP1 inflammasome activation in $\mathrm{AD}$ mice. Considering the importance of NLRP1 inflammasome-dependent pyroptosis in AD progression, we proposed that the mechanism underlying the neuroprotective effect of Bushen Huoxue Acupuncture may be associated with the inhibition of NLRP1 inflammasome-mediated pyroptosis. However, there are still many deficiencies in our study. For instance, NLRP3 inflammasome-mediated pyroptosis is also a crucial pyroptosis pathway, and plays an important role in $\mathrm{AD}$ development, and the effect of Bushen Huoxue Acupuncture on NLRP3 inflammasomemediated pyroptosis in $\mathrm{AD}$ is unknown. In addition, how the molecular mechanism of Bushen Huoxue Acupuncture 
suppresses NLRP1 inflammasome-mediated pyroptosis is also unclear. We will perform more experiments to explain these questions in further study.

\section{Conclusion}

In conclusion, our data demonstrated that Bushen Huoxue Acupuncture alleviated the cognitive deficit of the mouse AD model, inhibited $A \beta$ accumulation, and reduced neuronal apoptosis in the model. In addition, Bushen Huoxue Acupuncture possibly inhibits NLRP1 inflammasome-mediated pyroptosis to exert its neuroprotective effect. These results suggested that Bushen Huoxue Acupuncture may serve as a promising strategy for $\mathrm{AD}$ treatment.

\section{Acknowledgments}

This study was funded by The Famous of Old Chinese Medicine Experts Dong Ke-li’s Inheritance Studio Project.

\section{Disclosure}

The authors declare no conflicts of interest.

\section{References}

1. Jin Y, Sumsuzzman DM, Choi J, Kang H, Lee S-R, Hong Y. Molecular and functional interaction of the myokine irisin with physical exercise and Alzheimer's Disease. Molecules (Basel, Switzerland). 2018;23:3229. doi:10.3390/molecules23123229

2. Zhong L, Tong Y, Chuan J, Bai L, Shi J, Zhu Y. Protective effect of ethyl vanillin against $\mathrm{A} \beta$-induced neurotoxicity in PC12 cells via the reduction of oxidative stress and apoptosis. Exp Ther Med. 2019; 17:2666-2674.

3. Liao X, Cai F, Sun Z, et al. Identification of Alzheimer's diseaseassociated rare coding variants in the ECE2 gene. JCI Insight. 2020;5. doi:10.1172/jci.insight.135119

4. Jiang Z, Shen B, Xiang J. Metal-dependent interactions of metallothionein-3 beta-domain with amyloid-beta peptide and related physiological implications. J Inorg Biochem. 2019;196:110693. doi:10.1016/j.jinorgbio.2019.110693

5. Jin Y, Tu Q, Liu M. MicroRNA125b regulates Alzheimer's disease through SphK1 regulation. Mol Med Rep. 2018;18:2373-2380.

6. Yap JKY, Pickard BS, Chan EWL, Gan SY. The role of neuronal NLRP1 inflammasome in Alzheimer's disease: bringing neurons into the neuroinflammation game. Mol Neurobiol. 2019;56:7741-7753. doi:10.1007/s12035-019-1638-7

7. Chavarria-Smith J, Vance RE. The NLRP1 inflammasomes. Immunol Rev. 2015;265(1):22-34. doi:10.1111/imr.12283

8. Tan MS, Tan L, Jiang T, et al. Amyloid-beta induces NLRP1dependent neuronal pyroptosis in models of Alzheimer's disease. Cell Death Dis. 2014;5:e1382. doi:10.1038/cddis.2014.348

9. Xu TZ, Shen XY, Sun LL, et al. Ginsenoside Rg1 protects against $\mathrm{H} 2 \mathrm{O} 2$ induced neuronal damage due to inhibition of the NLRP1 inflammasome signalling pathway in hippocampal neurons in vitro. Int J Mol Med. 2019;43:717-726.

10. Lin C, Wei Z, Cheng -K-K, et al. (1)H NMR-based investigation of metabolic response to electro-acupuncture stimulation. Sci Rep. 2017;7:6820. doi:10.1038/s41598-017-07306-5
11. Li K, Giustini D, Seely D. A systematic review of acupuncture for chemotherapy-induced peripheral neuropathy. Curr Oncol. 2019;26: e147-e154.

12. Jia Y, Zhang X, Yu J, et al. Acupuncture for patients with mild to moderate Alzheimer's disease: a randomized controlled trial. BMC Complement Altern Med. 2017;17:556. doi:10.1186/s12906-017-2064-x

13. Cui S, Xu M, Huang J, Wang QM. Cerebral responses to acupuncture at GV24 and bilateral GB13 in rat models of Alzheimer's disease. Behav Neurol. 2018;2018.

14. Wang Z, Liang $P$, Zhao Z, et al. Acupuncture modulates resting state hippocampal functional connectivity in Alzheimer disease. PLoS One. 2014;9:e91160. doi:10.1371/journal.pone.0091160

15. Yu J, Zhang X, Liu C, Meng Y, Han J. Effect of acupuncture treatment on vascular dementia. Neurol Res. 2006;28:97-103. doi:10.1179/016164106X91951

16. Wang Z, Nie B, Li D, et al. Effect of acupuncture in mild cognitive impairment and Alzheimer disease: a functional MRI study. PLoS One. 2012;7:e42730. doi:10.1371/journal.pone.0042730

17. Zhang Z, Yang J, Liu C, et al. Pseudoginsenoside-F11 alleviates cognitive deficits and Alzheimer's disease-type pathologies in SAMP8 mice. Pharmacol Res. 2019;139:512-523. doi:10.1016/j. phrs.2018.10.024

18. Li Z. Experimental acupuncture. In: China Press of Traditional Chinese Medicine CoLtd. ISBN: 9787801564443. 2007.

19. Lin R, Chen J, Li X, et al. Electroacupuncture at the Baihui acupoint alleviates cognitive impairment and exerts neuroprotective effects by modulating the expression and processing of brain-derived neurotrophic factor in APP/PS1 transgenic mice. Mol Med Rep. 2016;13:1611-1617. doi:10.3892/mmr.2015.4751

20. Guo HD, Zhu J, Tian JX, et al. Electroacupuncture improves memory and protects neurons by regulation of the autophagy pathway in a rat model of Alzheimer's disease. Acupunct Med. 2016;34:449-456. doi:10.1136/acupmed-2015-010894

21. Blennow K, Zetterberg H. Biomarkers for Alzheimer's disease: current status and prospects for the future. $J$ Intern Med. 2018;284:643-663. doi:10.1111/joim.12816

22. Nakagami Y, Nishimura S, Murasugi T, et al. A novel beta-sheet breaker, RS-0406, reverses amyloid beta-induced cytotoxicity and impairment of long-term potentiation in vitro. $\mathrm{Br} J$ Pharmacol. 2002;137:676-682. doi:10.1038/sj.bjp.0704911

23. Widenbrant MJO, Rajadas J, Sutardja C, Fuller GG. Lipid-induced beta-amyloid peptide assemblage fragmentation. Biophys $J$. 2006;91:4071-4080. doi:10.1529/biophysj.106.085944

24. Pontillo A, Catamo E, Arosio B, Mari D, Crovella S. NALP1/NLRP1 genetic variants are associated with Alzheimer disease. Alzheimer Dis Assoc Disord. 2012;26:277-281. doi:10.1097/WAD.0b013e318231a8ac

25. Saresella M, La Rosa F, Piancone F, et al. The NLRP3 and NLRP1 inflammasomes are activated in Alzheimer's disease. Mol Neurodegener. 2016;11:23. doi:10.1186/s13024-016-0088-1

26. Kollmer M, Close W, Funk L, et al. Cryo-EM structure and polymorphism of A $\beta$ amyloid fibrils purified from Alzheimer's brain tissue. Nat Commun. 2019;10:4760. doi:10.1038/s41467-019-12683-8

27. Hu Z, Yang Y, Gao K, Rudd JA, Fang M. Ovarian hormones ameliorate memory impairment, cholinergic deficit, neuronal apoptosis and astrogliosis in a rat model of Alzheimer's disease. Exp Ther Med. 2016;11:89-97. doi:10.3892/etm.2015.2868

28. Zhang B, Zhang Y, Wu W, et al. Chronic glucocorticoid exposure activates BK-NLRP1 signal involving in hippocampal neuron damage. J Neuroinflammation. 2017;14:139.

29. An Z, Wang D, Yang G, Zhang WQ, Ren J, Fu JL. Role of microRNA-130a in the pathogeneses of obstructive sleep apnea hypopnea syndrome-associated pulmonary hypertension by targeting the GAX gene. Medicine (Baltimore). 2017;96:e6746.

30. Shi J, Gao W, Shao F. Pyroptosis: gasdermin-mediated programmed necrotic cell death. Trends Biochem Sci. 2017;42:245-254. doi:10.1016/j.tibs.2016.10.004 


\section{Publish your work in this journal}

Neuropsychiatric Disease and Treatment is an international, peerreviewed journal of clinical therapeutics and pharmacology focusing on concise rapid reporting of clinical or pre-clinical studies on a range of neuropsychiatric and neurological disorders. This journal is indexed on PubMed Central, the 'PsycINFO' database and CAS, and

is the official journal of The International Neuropsychiatric Association (INA). The manuscript management system is completely online and includes a very quick and fair peer-review system, which is all easy to use. Visit http://www.dovepress.com/testimonials.php to read real quotes from published authors. 